\title{
Power in isolation: insights from single cells
}

\section{Benjamin J. Cole, Jonelle T. R. Basso and Axel Visel}

This month's Genome Watch highlights a set of single-cell approaches that yield powerful insights into microbial biology.

Although genome sequencing of microorganisms is now routine, deciphering how genomic content relates to organismal, environmental and ecological processes remains a grand challenge. A set of recent studies successfully applied an array of single-cell approaches to link microbial genes and genomes to function.

In one study, Doud and colleagues ${ }^{1}$ developed a single-cell genome sequencing strategy to identify cellulose-degrading microorganisms in a complex community. They first introduced fluorescently labeled cellulose particles as 'bait' into samples taken from a natural aquatic environment. Then, using fluorescence-activated cell sorting (FACS) and whole-genome amplification, they devised a targeted approach to fish out microorganisms of interest that were bound to the substrate, which suggests that they are likely to degrade cellulose. Sequencing individual genomes of the captured cellulose-bound microbial cells revealed a rare biosphere member of the poorly characterized clade Goldbacteria, laden with putative cellulase genes. The authors then functionally validated the activity of cellulose-degrading enzymes within this novel strain named Candidatus 'Cellulosimonas argentiregionis', which will assist with annotating similar genes in rapidly growing bacterial genome data repositories.

Although this approach powerfully identifies unknown microorganisms with a desired function within a complex community, it hinges on sequence comparisons with previously characterized genes and genomes for functional interpretation. To address this challenge, Thibault and colleagues ${ }^{2}$ devised a complementary single cell-based method by leveraging recently developed microfluidics techniques to enable droplet transposon sequencing (dTn-Seq). Unlike traditional Tn-Seq, whereby insertion mutants are grown as pooled libraries under control and altered conditions, dTn-Seq enables hundreds of thousands of individual mutants to be captured in microdroplets and grown in isolation. This removes a confounding factor often observed in bulk culture Tn-Seq approaches, where phenotypes of individual mutants may be suppressed by the presence of other strains in the community. For example, a mutation in a gene required for producing a secreted quorum sensing factor may affect the population dynamics of a mutant strain grown in isolation but will be undetectable in liquid co-culture with many other mutants. Thibault et al. used $\mathrm{dTn}$-Seq to identify phenotypes of thousands of Streptococcus pneumoniae mutants. When mutants were grown in droplets, fitness contributions of many genes became apparent that would have been missed if grown together. This technology is compatible with insertion sequencing (IN-seq), FACS, transposondirected insertion site sequencing (Tra-DIS) and high-throughput insertion tracking by deep sequencing (HITS), which enables its application in both bacteria and unicellular eukaryotes, such as yeasts. It can also be combined with microscopy and double encapsulation for interactive screening with other microbial strains or species present

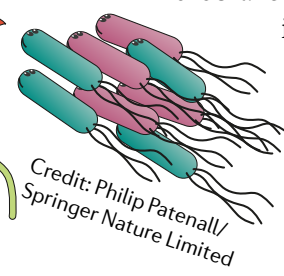
in the microdroplets.

Genetics and genomics methods successfully establish general relationships between microbial genes and functional traits. However, more quantitative insights into the role of genes and gene regulatory networks typically require functional genomics methods, such as transcribed RNA measurements. Single-cell RNA sequencing (scRNA-seq) combines microfluidic cell isolation with transcriptome barcoding and sequencing. It is now routinely used in studies of multicellular organisms, but it is also increasingly applied in unicellular eukaryotes. For example, scRNA-seq studies of pathogenic Plasmodium and Toxoplasma species have provided a direct window into the transcriptome landscapes of their parasitic life cycles. These deconvoluted views of transcriptional heterogeneity across individual cells revealed novel cellular subclusters and transition points, thus uncovering important leads for understanding cell cycle progression and virulence ${ }^{3-5}$. The application of similar methods to bacteria and archaea is not yet possible because established scRNA-seq protocols typically rely on the extended polyadenylated transcript tails found in eukaryotes. However, as technology progresses, similar methods are expected to become available for bacteria and archaea, holding equally great promise to unlock untold secrets of single cells.

$$
\begin{aligned}
& \text { Benjamin J. Cole }{ }^{凶} \text {, Jonelle T. R. Basso } \\
& \text { and Axel Visel }{ }^{\square}
\end{aligned}
$$

Doud, D. F. R. et al. ISME J. 14, 659-675 (2019).

Thibault, D. et al. Nat. Commun. 10, 5729 (2019).

3. Walzer, K. A. et al. PLoS Genet. 15, e 1008506

(2019).

4. Xue, Y. et al. eLife 9, e54129 (2020)

5. Howick, V. M. et al. Science 365, (2019).

\section{Acknowledgements}

The U.S. Department of Energy Joint Genome Institute, a DOE Office of Science User Facility, is supported under Contract No. DE-AC02-05CH11231.

\section{Competing interests}

The authors declare no competing interests. 